Reprod. Nutr. Dévelop., 1983, 23 (6), 959-965.

\title{
Étude de la gastrine chez Gallus gallus. II. - Impossibilité de détecter une immunoréactivité de type gastrine dans le sérum des poulets
}

\author{
Nicole RIDEAU, P. MONGIN
}

Station de Recherches avicoles, I.N.R.A. Nouzilly 37380 Monnaie, France.

Summary. A study of gastrin in Gallus gallus. II. No evidence of gastrin-like immunoreactivity in chicken serum.

Antihuman gastrin 1.17 antibodies capable of at least partly detecting gastrin-like components in avian pyloric mucosa did not permit the measurement of circulating gastrin either in the basal or the stimulated state or after in vitro extraction and concentration of the sera. Human gastrin 1.17 was quantitatively recovered in avian serum but tissular gastrin-like immunoreactivity doubled after in vitro incubation of avian pyloric extracts in the serum. It is proposed that the circulating gastrin-like component(s) was poorly recognized in the present assay and so escaped measurement; alternatively, gastrin might be released in, or rapidly converted into, a smaller form than heptadecapeptide gastrin.

\section{Introduction.}

Nous avons montré dans une note précédente (Rideau et Mongin, 1983) qu'un anticorps antigastrine humaine 1.17 reconnaît dans des extraits de jonction pylorique de poulet (qui possèdent par ailleurs une activité gastrosecrétoire chez le poulet) un ensemble de 3 substances de poids moléculaire supérieur ou égal à celui de la gastrine humaine à 17 acides aminés. II n'existe actuellement aucune donnée bibliographique concernant la gastrine circulante des oiseaux; c'est pourquoi nous avons recherché, à l'aide de ce même anticorps, s'il est possible de mettre en évidence une gastrine immunoréactive dans le sang des poulets à l'état basal et sous diverses stimulations physiologiques. 


\section{Matériel et méthodes}

On utilise pour le dosage radioimmunologique de la gastrine sérique le même matériel que celui décrit pour les extraits tissulaires (trousse Gask CEA, Saclay France) (Rideau et Mongin, 1983). Nous rappelons que le pourcentage de réaction croisée de l'anticorps antigastrine humaine 1.17 est respectivement de 100, $73,30,3,5$ et 3,5 pour les gastrines humaines 1.17 et 2.17 , la big gastrin, la cholecystokinine et la pentagastrine (trousse Gask CEA). Les essais sont effectués sur sérum ou sur plasma (en présence de 5 unités d'héparine par $\mathrm{ml}$ de sang, qui n'interfèrent pas avec la réaction immunologique).

\section{A. Traitement des sérums.}

Les sérums de poulet ainsi que des solutions témoins de gastrine humaine 1.17 et d'extraits pyloriques aviaires (en tampon veronal $0,02 \mathrm{M} \mathrm{pH} \mathrm{8,4)} \mathrm{ont} \mathrm{été}$ soumis aux traitements suivants :

- Adsorption sur charbon Norit-A $\left(200 \mathrm{mg} / \mathrm{ml}\right.$ de sérum) $30 \mathrm{~min}$ à $20^{\circ} \mathrm{C}$ sous agitation, suivie de 3 centrifugations à $2000 \mathrm{~g}(1 \mathrm{~h})$ afin d'éliminer toute trace de charbon; ce traitement élimine plus de $99 \%$ de la gastrine iodée radioactive ajoutée au sérum et ne modifie pas significativement sa teneur en protéines (Kumar et Deodhar, 1975).

- Résistance à l'incubation $10 \mathrm{~min}$ à $100^{\circ} \mathrm{C}$ (bain-marie bouillant) ; les surnageants de centrifugation sont répartis en deux fractions : l'une est stockée telle quelle à $-20^{\circ} \mathrm{C}$, l'autre après lyophilisation, est dissoute dans un volume variable de tampon veronal $0,02 \mathrm{M}, \mathrm{pH} \mathrm{8,4}$, ce qui permet une concentration des échantillons de deux à huit fois.

- Extraction par l'éthanol à $80 \%$, évaporation et dissolution dans le même tampon que précédemment (concentration de 1 à 8 fois).

- Incubation en présence de trypsine $10,25 \mathrm{mg} / \mathrm{ml}$ de sérum) suivie d'une incubation de $10 \mathrm{~min}$ à $100^{\circ} \mathrm{C}$ au bain-marie bouillant afin d'inactiver l'enzyme (Rehfeld, 1972).

- Récupération en milieu sérique de la gastrine immunoréactive présente dans les solutions témoins : les échantillons sont ajoutés à du sérum de poulet fraîchement prélevé, en présence ou non d'aprotinine $(1800 \mathrm{KIU} / \mathrm{ml})$ et à du sérum incubé pendant $10 \mathrm{~min}$ à $100^{\circ} \mathrm{C}$ (bain-marie bouillant).

Chaque traitement a été répété sur plusieurs sérums et pour les solutions témoins, sur deux dilutions différentes ; chaque échantillon est dosé en double.

\section{B. Stimulation physiologique.}

1. Gavage. - L'essai a été effectué sur des poulets mâles (souche Hubbard) âgés de 5 semaines. Ces animaux à jeun depuis $18 \mathrm{~h}$ reçoivent par gavage à l'aide d'un catheter introduit dans le jabot un mélange constitué d'une partie d'un aliment équilibré à $20 \%$ de protéines pour deux parties d'eau correspondant en quantité au $1 / 7$ ou $1 / 5$ de la ration quotidienne. Du sang est prélevé par voie intracardiaque sur 7 animaux à chaque temps (toutes les $3 \mathrm{~min}$ puis aux temps 20,30 et $80 \mathrm{~min}$ après le gavage, un seul prélèvement par poulet), les plasmas sont rapidement décantés et conservés à $-20^{\circ} \mathrm{C}$. 
2. Perfusion de Bombésine (BBS). - $10 \mathrm{ng} / \mathrm{kg} / \mathrm{mn}$ de Bombésine sont perfusés à 3 poules pondeuses (souche Warren) anesthésiées à l'aide de Fluothane. Les poules sont munies d'un catheter de perfusion (veine allaire) et de 2 catheters de prélèvement (veine allaire et veine pancréaticoduodénale pour les prises de sang veineux périphérique et antral). Les prélèvements sont effectués aux temps $0,5,10$ et 20 min après le début de la perfusion. Dans une manipulation parallèle on a vérifié que la dose de BBS utilisée provoque dans les 5 min qui suivent le début de la perfusion une stimulation intense de la secrétion gastrique d'acide chez lä poule pondeuse anesthésiée.

Les produits chimiques utilisés sont les suivants : charbon Norit-A (U. S. Biochemical Co.) : aprotinine (Sigma A-6012); Bombesine (Farmitalia 20087) ; trousse Gask pour le dosage radioimmunologique de la gastrine humaine (CEASORIN, Saclay, France).

\section{Résultats.}

1. Les quantités de substance immunoréactive mesurées dans les sérums de poulet en dehors de toute stimulation (en présence ou non d'aprotinine (1800 $\mathrm{Kiu} / \mathrm{ml}$ )) oscillent autour de $10 \mathrm{pg}$ équivalents gastrine humaine $1.17 \mathrm{par} \mathrm{ml}$ soit 3 à 4 fois le seuil de sensibilité du dosage (Rideau et Mongin, 1983). Après ébullition des sérums, on ne détecte plus d'immunoréactivité (tabl. 1) tandis qu'une adsorption sur charbon, plusieurs décongélations successives ou un jeûne pro-

\section{TABLEAU 1}

Récupération et concentration de gastrine immunoréactive 1.17 après différents traitements physicochimiques des sérums

Traitement

\begin{tabular}{|c|c|c|c|c|c|c|c|c|}
\hline \multirow{2}{*}{$\begin{array}{l}\text { Gastrine immunoréactive } \\
\text { (pg équivalents } \\
\text { gastrine humaine } 1.17 / \mathrm{ml} \text { ) }\end{array}$} & \multicolumn{3}{|c|}{$\begin{array}{l}\text { Bain-marie bouillant } \\
\text { (10 min) }\end{array}$} & \multicolumn{3}{|c|}{$\begin{array}{l}\text { Extraction par } \\
\text { de l'éthanol à } 80 \%\end{array}$} & \multicolumn{2}{|c|}{$\begin{array}{l}\text { Digestion trypsique } \\
\left(10 \mathrm{~min} \text { à } 37^{\circ} \mathrm{C}\right)\end{array}$} \\
\hline & $A(1)$ & B & C & A & $\mathrm{B}$ & $\mathrm{C}$ & A & $\mathrm{B}$ \\
\hline Gastrine humaine $1.17(3)$ & $\begin{array}{r}42 \\
106\end{array}$ & $\begin{array}{l}55 \\
99\end{array}$ & $\begin{array}{l}94(110)(2) \\
160(190)\end{array}$ & $\begin{array}{l}18 \\
62\end{array}$ & $\begin{array}{l}22 \\
72\end{array}$ & $\begin{array}{c}85(65) \\
-\end{array}$ & $\begin{array}{l}33 \\
70\end{array}$ & $\begin{array}{l}33 \\
58\end{array}$ \\
\hline $\begin{array}{l}\text { Extrait de jonction pylorique } \\
\text { aviaire }\end{array}$ & $\begin{array}{r}30 \\
148\end{array}$ & $\begin{array}{r}40 \\
137\end{array}$ & $\begin{array}{l}141(200) \\
235(275)\end{array}$ & $\begin{array}{r}37 \\
150\end{array}$ & $\begin{array}{r}44 \\
136\end{array}$ & $\begin{array}{c}80(100) \\
-\end{array}$ & $\begin{array}{r}38 \\
171\end{array}$ & $\begin{array}{r}78 \\
274\end{array}$ \\
\hline Sérum de poulet & $\begin{array}{l}9 \\
6 \\
7\end{array}$ & $\begin{array}{l}0 \\
0 \\
0\end{array}$ & $\begin{array}{l}3(\times 5) \\
3(\times 7) \\
8(\times 2)\end{array}$ & $\begin{array}{l}4 \\
9 \\
8\end{array}$ & $\begin{array}{l}0 \\
0 \\
0\end{array}$ & $\begin{array}{r}4(\times 4) \\
8(\times 6) \\
10(\times 6)\end{array}$ & $\begin{array}{l}8 \\
7\end{array}$ & $\begin{array}{l}0 \\
0\end{array}$ \\
\hline
\end{tabular}

(1) A : Valeur avant traitement,

B : Valeur après traitement et reprise quantitative par le tampon veronal, nal).

$\mathrm{C}$ : Valeur après traitement et concentration (reprise par un volume moindre de tampon vero-

(2) Entre parenthèses : valeur attendue après concentration-ou pour les sérums, facteur de concentration.

(3) Gastrine humaine 1.17 en tampon veronal $0.02 \mathrm{M} \mathrm{pH} \mathrm{8,4.}$ 
longé ( 6 jours) ne modifient pas l'immunoréactivité initialement détectée dans les sérums, ce qui signifierait qu'à l'état basal la substance dosée dans ce système ne représenterait pas réellement de la gastrine.

Les essais de concentration (de 2 à 8 fois) après extraction par l'éthanol à $80 \%$ ou ébullition $10 \mathrm{~min}$ au bain-marie bouillant sont possibles pour les solutions témoins de gastrine humaine 1.17 ou d'extrait antral aviaire mais ne permettent pas d'augmenter la concentration en équivalent gastrine dans les sérums de poulet (tabl. 1). En outre, l'incubation des sérums avec une solution de trypsine ne fait pas apparaître de forme moléculaire nouvelle qui serait mieux reconnue par les anticorps utilisés, comme on l'observe pour les extraits pyloriques (tabl. 1) dans lesquels l'augmentation de l'immunoréactivité mesurée s'explique par l'apparition de la forme à 17 acides aminés (Rideau et Mongin, 1983). La gastrine humaine 1.17 n'est pas détruite après incubation avec du sérum ou du sang de poulet, sa récupération varie entre 95 et $100 \%$, tandis que la substance immunoréactive présente dans la jonction pylorique de poulet $n^{\prime}$ est retrouvée quantitativement qu'après incubation avec des sérums aviaires préalablement traités $10 \mathrm{~min}$ à $100^{\circ} \mathrm{C}$ (tabl. 2) : les valeurs retrouvées après incubation des extraits dans du sérum frais sont en effet 2 fois plus importantes qu'après incubation dans le tampon véronal ou dans du sérum bouilli.

2. Après des stimulations physiologiques connues pour augmenter la gastrinémie des mammifères : perfusion de BBS (Bertaccini et al., 1971), à une dose qui stimule la sécrétion gastrique des poulets (Linari et al., 1975) ; distribution par gavage d'un repas constitué d'un aliment équilibré à $20 \%$ de protéines (Dockray et Taylor, 1976), on constate que I'anticorps utilisé ne détecte pas d'augmentation de l'immunoréactivité sérique au niveau périphérique ou dans le sang veineux antral ; les valeurs mesurées à ce niveau (pour l'essai BBS) sont toutefois toujours supérieures aux valeurs systémiques (tabl. 3).

\section{TABLEAU 2}

Comportement de la gastrine (humaine et aviaire) en milieu sérum de poulet (anticorps antigastrine humaine 1.17)

\begin{tabular}{|c|c|c|c|c|}
\hline \multicolumn{5}{|c|}{ Traitement } \\
\hline \multirow[b]{2}{*}{$\begin{array}{c}\text { Gastrine } \\
\text { immunoreactive } \\
\text { (pg équivalents } / \mathrm{ml} \text { ) }\end{array}$} & \multirow{2}{*}{$\begin{array}{c}\text { Solution } \\
\text { de départ } \\
\text { en tampon veronal } \\
0.02 \mathrm{M} / \mathrm{pH} \mathbf{8 , 4}\end{array}$} & \multicolumn{3}{|c|}{ Additionnée à } \\
\hline & & $\begin{array}{l}\text { sérum } \\
\text { de poulet }\end{array}$ & $\begin{array}{c}\text { sérum } \\
\text { de poulet } \\
+ \text { Aprotinine } \\
1800 \mathrm{Kiu} / \mathrm{ml})\end{array}$ & $\begin{array}{c}\text { sérum } \\
\text { de poulet } \\
\text { bouilli } \\
\text { (10 mn BMB) }\end{array}$ \\
\hline Gastrine humaine 1.17 & $\begin{array}{l}52 \\
75\end{array}$ & $\begin{array}{l}58(62)\left(^{*}\right) \\
89(90)\end{array}$ & $\begin{array}{l}53(62) \\
78(90)\end{array}$ & $\begin{array}{c}55(52) \\
-\end{array}$ \\
\hline $\begin{array}{l}\text { Extrait de jonction } \\
\text { pylorique aviaire }\end{array}$ & $\begin{array}{r}18 \\
100 \\
45 \\
70\end{array}$ & $\begin{aligned} 59(24) \\
227(124) \\
95(48) \\
180(85)\end{aligned}$ & $\begin{array}{c}62(24) \\
213(124) \\
- \\
-\end{array}$ & $\begin{array}{c}- \\
\overline{-} \\
58(45) \\
79(70)\end{array}$ \\
\hline
\end{tabular}

(*) Entre parenthèses : valeur attendue si l'on tient compte de l'immunoréactivité endogène des sérums de poulet. 
TABLEAU 3

Stimulation physiologique de la gastrinémie (pg équiv. gastrine humaine $1.17 / \mathrm{ml}$ )

a) Gavage (*) de poulets mâles de 5 semaines

\begin{tabular}{cccccccccc}
\hline $\begin{array}{c}\mathrm{t} \\
\text { (min après gavage) }\end{array}$ & 0 & 3 & 6 & 9 & 12 & 15 & 20 & 30 & 80 \\
\hline $\begin{array}{c}\overline{\mathrm{x}} \\
(\mathrm{pg} / \mathrm{ml})\end{array}$ & 12,6 & 12,8 & 9,1 & 11,1 & 10,1 & 12,3 & 15,3 & 12 & 12,9 \\
\hline $\mathrm{s} \overline{\mathrm{x}}$ & 3,2 & $\mathbf{1 , 0}$ & $\begin{array}{l}0,6 \\
\mathrm{n}\end{array}$ & $\begin{array}{l}1,2 \\
6\end{array}$ & $\begin{array}{l}0,6 \\
7\end{array}$ & $\begin{array}{l}7,9 \\
7\end{array}$ & $\begin{array}{l}3,3 \\
7\end{array}$ & $\begin{array}{l}1,8 \\
7\end{array}$ & $\begin{array}{l}0,6 \\
5\end{array}$ \\
\hline
\end{tabular}

${ }^{*}{ }^{*} 1 / 7$ de la quantité journalière (ration équilibrée à $20 \%$ de protéines) - prise de sang intracardiaque -5 à 7 poulets par temps:

b) Perfusion de Bombesine (*); prélèvement de sang veineux antral et périphérique

\begin{tabular}{crrrr}
\hline$t$ & \multicolumn{1}{c}{10} & \multicolumn{1}{c}{20} \\
(min de perfusion) & 0 & 5 & 10 & \\
\hline sg.v. périph. $(\mathrm{pg} / \mathrm{ml})$ & $7,3 \pm 0,7$ & $6,5 \pm 1,5$ & $6,7 \pm 1,2$ & $6,7 \pm 1,2$ \\
sg.v. antral $(\mathrm{pg} / \mathrm{ml})$ & $10+ \pm 1,5$ & $12,5 \pm 8,5$ & $11,7 \pm 2,3$ & $9,7 \pm 0,7$ \\
\hline
\end{tabular}

(*) $10 \mathrm{ng} / \mathrm{kg} / \mathrm{mn}$ Bombesine après $\mathrm{t}=0$ - poules pondeuses anesthésiées $\mathrm{n}=3$ (sauf $\mathrm{t}=5 \mathrm{mn}: \mathrm{n}=2$ ).

L'absence de stimulation de la gastrine circulante après un repas équilibré ou après un repas constitué de protéines de soja pures $(5 \mathrm{~g} / \mathrm{kg})$ a été confirmée avec un autre anticorps (anticorps $28 \mathrm{C}$, don du Docteur J. A. Chayvialle). Cet anticorps qui reconnaît de façon équivalente les gastrines humaines à 17 acides aminés et à 14 acides aminés (Chayvialle et al., 1978) reconnaît également dans la muqueuse pylorique de poulet un ensemble de 3 molécules dont le comportement immunologique et chromatographique est superposable à celui décrit précédemment (Rideau et Mongin, 1983).

\section{Discussion.}

Les faibles taux de substance immunoréactive mesurés dans le sang des poulets ne résultent pas d'une dégradation de gastrine (1.17) in vitro par des enzymes protéolytiques sériques puisque l'aprotinine ajouté à du sérum ou à du sang total de poulet ne modifie pas les taux mesurés; en outre la gastrine humaine 1.17 exogène est stable dans ce milieu. En revanche, l'impossibilité d'obtenir des sérums de poulet exempts de gastrine à la suite d'un jeûne prolongé (Lichtenberger et al., 1975) d'adsorption sur charbon, ou de décongélations successives 
(Bryant, 1978), et la perte d'immunoréactivité après ébullition, montrent que les $10 \mathrm{pg} / \mathrm{ml}$ mesurés en moyenne résultent d'un artefact de dosage. Celui-ci pourrait être lié à l'établissement de la courbe d'étalonnage en milieu tamponné sans sérum aviaire " dégastriné ". II s'en suit que l'on ne peut pas mesurer les quantités absolues de gastrine immunoréactive à l'état basal dans les sérums de poulet. Les essais réalisés par la suite visant à extraire la gastrine circulante de poulet et/ou à la concentrer se sont avérés infructueux ; les épreuves de stimulation destinées à augmenter la gastrine circulante n'ont pas permis non plus de détecter les gastrinémies élevées attendues.

Chez les mammifères, la gastrine, comme la plupart des hormones peptidiques, présente une forte hétérogénéité moléculaire et l'on a pu montrer que la distribution relative de ses différents constituants varie considérablement selon les milieux biologiques, les espèces et l'état physiologique considéré. Cette hétérogénéité moléculaire a de sérieuses conséquences pour l'extraction, la mesure et l'évaluation biologique de la gastrine puisqu'une différence de taille, de charge ou la substitution d'un seul acide aminé modifie les propriétés physiques, immunologiques et physiologiques de la molécule (Rehfeld, 1980). Elle nécessite pour le problème particulier de la détection immunologique de la gastrine circulante de disposer de plusieurs antisérums de spécificités variées puisque l'on sait que chez les mammifères, les formes de stockage antral de la gastrine diffèrent des formes circulantes $(*)$.

A la suite de notre étude il est possible d'envisager deux hypothèses permettant d'expliquer l'impossibilité de détecter une véritable gastrinémie basale ou après des stimulations connues pour augmenter, chez les mammifères, la gastrine sérique : D'une part la spécificité de l'antisérum utilisé n'est connue que pour les formes humaines de la gastrine et il est possible que l'anticorps ne reconnaisse pas quantitativement ces mêmes formes aviaires. Une réactivité faible pourrait ainsi expliquer à la fois les valeurs basses d'immunoréactivité dans le tissu antropylorique (Rideau et Mongin, 1983) et l'impossibilité de doser la gastrine sérique. $D^{\prime}$ autre part les taux de récupération supérieurs à $100 \%$ observés après incubation d'extrait pylorique de poulet dans du sérum de poulet mettent en évidence une conversion possible des formes tissulaires par le sérum lui-même (tabl. 2). Ces taux ne proviennent pas d'une altération du traceur qui rendrait impossible sa liaison à l'anticorps (la gastrine humaine 1.17 est retrouvée quantitativement dans des conditions d'incubation analogues), ils semblent plutôt indiquer qu'il existe dans le sérum de poulet une substance (thermolabile et résistante à 1800 Kiu aprotinine par $\mathrm{ml}$ de sérum) capable de transformer la gastrine tissulaire

(*) La gastrine à 17 acides aminés prédomine $(>90 \%)$ dans la muqueuse antrale de l'homme, du porc, du chien et du chat (Rehfeld et Uvnäs-Wallensten, 1978 ; Dockray et al., 1975 ; Rehfeld et al., 1975) tandis que pour les trois premières espèces citées, les $2 / 3$ de la gastrine circulante sont constituées, en période de jeûne, de gastrine à 34 acides aminés ; après un repas, cette forme ne représente plus que la moitié de la gastrine totale circulante, l'autre moitié étant principalement constituée de gastrine à 17 acides aminés ainsi que de quantités minimes de gastrine à 14 acides aminés et de composant I (progastrine). A l'opposé, chez le chat, la gastrine à 34 acides aminés n'est pas libérée dans la circulation sanguine et l'on retrouve dans ce milieu des quantités importantes de gastrine à 14 acides aminés provenant de la dégradation de gastrine 1.17 (Rehfeld et Uvnäs-Wallensten, 1978). 
en une (ou plusieurs) formes - I'une d'entre elles au moins est stable in vitro et reconnue par les anticorps utilisés. In vivo un catabolisme plus poussé pourrait transformer la molécule de gastrine à 17 acides aminés en une molécule de poids moléculaire inférieur. Comme chez le chat, la gastrine du poulet pourrait donc être sécrétée ou rapidement transformée en une " mini gastrine » non reconnue par l'antisérum utilisé.

Reçu en octobre 1982. Accepté en mai 1983.

Remerciements. - Nous remercions vivement le Docteur J. A. Chayvialle pour son aimable don d'anticorps antigastrine humaine 1.17 (anticorps $28 \mathrm{C}$ ).

\section{Références}

BERTACCINI G., ERPSAME V., MELCHIORRY P., SOPRANZI N., 1971. Gastrin release by bombesin in the dog. Br. J. Pharmacol., 52, 219-225.

BLAIR E. L., HARPER A. A., LAKE H. J., REED W. D., SCKATCHERD T., 1961. A simple method of preparing gastrin. J. Physiol. (Lond.), 156, 11-12.

BRYANT M. G., 1978. Plasma artefacts in radioimmunoassay, 120-125. In S. R. BLOOM, Gut hormones. Churchill Livingstone, Edinburgh, London and New York.

CHAYVIALLE J. A. P., DESCOS F., BERNARD C., MARTIN A., BARBÉ C., PARTENSKY C., 1978. Somatostatin in mucosa of stomach and duodenum in gastrointestinal disease. Gastroenterology, 75, 13-19.

DOCKRAY G. I., DEBAS H. T., WALSH I. H., GROSSMAN M. I., 1975. Molecular forms of gastrin in antral mucosa and serum of dogs. Proc. Soc. exp. Biol. Med., 149, 550-554.

DOCKRAY C. J., TAYLOR I. L., 1976. Heptadecapeptide gastrin : measurement in blood by specific radioimmunoassay. Gastroenterology, 71, 971-977.

KUMAR M. S., DEODHAR J. D., 1975. An improved radioimmunoassay of serum gastrin using a commercial kit. Clin. chim. Acta, 61, 191-197.

LICHTENBERGER L. M., LECHAGO J., JOHNSON L. R., 1975. Repression of antral and serum gastrin concentration by food deprivation in the rat. Gastroenterology, 68, 1473-1479.

LINARI G., BALDIERI M. and ANGELUCCI L., 1975. The action of bombesin on gastric secretion of the chicken. Bur. J. Pharmacol., 34, 143-150.

REHFELD J. F., 1972. Three components of gastrin in human serum. Gel filtration studies on the molecular size of immunoreactive serum gastrin. Bioch. biophys. Acta, 285, 363-372.

REHFELD J. F., 1980. Heterogeneity of gastrointestinal hormones, 433-449. In G. B. JERZY GLASS, Gastrointestinal hormones. Raven Press, New York.

REHFELD J. F., STADIL F., MALMSTRÖM J., MIYATA M., 1975. Gastrin heterogenety in serum and tissue, 43-58. In J. C. THOMPSON, Gastrointestinal hormones. Univ. Texas Press, Austin and London.

REHFELD J. F., UVNÄS-WALLENSTEN K., 1978. Gastrin in cat and dog: evidence for a biosynthetic relationship between the large molecular forms of gastrin and heptadecapeptide gastrin. J. Physiol. (Lond.), 283, 379-396.

RIDEAU N., MONGIN P., 1983. Etude de la gastrine chez Gallus gallus. I. Mise en évidence d'une immunoréactivité et d'une activité biologique de type gastrine dans le tractus digestif de poulets. Reprod. Nutr. Dévelop., 23, 793-941. 